\section{Toothpaste tablets: the future of toothpaste}

High quality, environmentally friendly with a fresh clean feeling: this is what patients want from their toothpaste tablet. No longer do you have to compromise between protecting your oral health and protecting our planet.

With Tongue's peppermint toothpaste tablets, your patients can feel good about protecting their oral health with 1450 ppm fluoride and protecting our planet with one less plastic toothpaste tube ending up in landfill.

Tongue's toothpaste tablet pouches are home compostable and delivered direct to your door through your letterbox, making the switch from traditional toothpaste to toothpaste tablets simple, easy and cost effective.

Simply chew the toothpaste tablet for a few seconds, moisten your manual or electric toothbrush bristles with water and brush your teeth as normal. You can also add a compostable bamboo toothbrush with replaceable heads to your order for the full eco-friendly dental experience.

Tongue's was founded by UK qualified dentists with the mission of helping to put an end to the plastic crisis in dentistry. They wanted to develop a range of eco-friendly dental products which they would feel confident recommending to their patients without any compromise to their patients' oral health.

For more information visit www. tonguesclub.com. If you are interested in stocking Tongue's eco-friendly dental products in your practice, contact hello@tonguesclub.com.

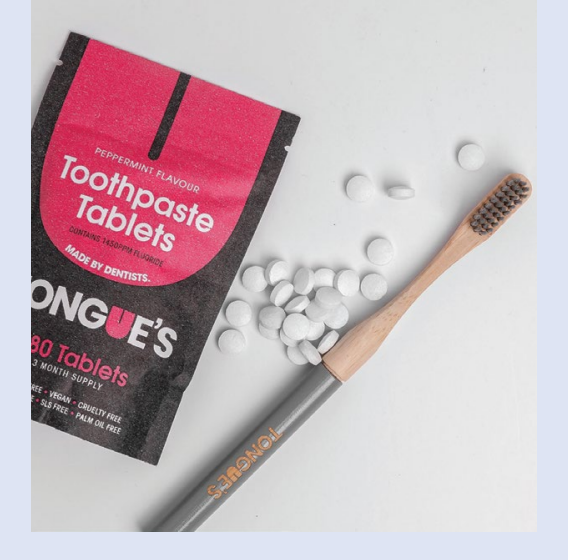

\title{
App uses artificial intelligence to soothe patients with music
}

Patients around the world will soon be prescribed soothing music chosen by artificial intelligence to ease their anxiety and pain, thanks to an encouraging trial of a health tech app on dementia patients.

British health tech start-up MediMusic has created an app and a streaming device called the MediBeat that dispenses personalised playlists to reduce anxiety and pain in patients using a 'digital drip' to administer the most calming music.

It could revolutionise the treatment of dementia, pre/post operation, chronic pain, dentistry, and Alzheimer's Disease through to improving motor response as part of a physical rehabilitation programme.

Initial clinical NHS trials at Lancashire Teaching Hospitals NHS Foundation Trust found the use of MediMusic saw an up to $22 \%$ reduction in heart rate in patients with dementia during the COVID-19 pandemic. It can be used in hospitals, care homes and dentist surgeries and could slash medication bills by up to a quarter.

Music therapy has already been proven in several previous studies to reduce anxiety by $44 \%$ and pain by $28 \%$. This has resulted in a reduction in the need for relevant medication by $24 \%{ }^{1}$

Now the revolutionary treatment is undergoing an NHS trial also at Lancashire Teaching Hospitals NHS Foundation Trust, on 40 NHS doctors and nurses and other staff who have worked in critical care during the pandemic, to help them ease anxiety and stress.

The MediMusic app works with the patient's age, gender, nationality, and ethnicity and based on sociological and psychological science, it then compiles in seconds the perfect 20-minute playlist of soothing music to calm them.

Playlist running order is designed to reduce heart rate and stress hormones like cortisol and promotes relaxation through hormones like dopamine and oxytocin. The music is played through earphones and the MediBeat streaming device and a heart rate monitor worn on the wrist. The brain responds to music more than any other stimulus. MediMusic's proprietary algorithms extract the relevant features from the digital DNA of a piece of music, resulting in a fingerprint for healthcare use.

Using artificial intelligence, machine learning and the data about the patient, MediMusic then automatically creates playlists from music streaming services within a couple of clicks and plays the music through a streaming device called the MediBeat and a pair of headphones. Each chosen track is 'heartrate optimised' to reduce anxiety, stress or pain, improve quality of life and streamline healthcare workflow.

A heart rate monitor worn on the wrist allows MediMusic to monitor the physiological effect of a piece of music upon a listener and if the listener's heart rate does not respond as expected, MediMusic's 'Digital Drip' uses artificial intelligence and machine learning to swap out forthcoming playlist tracks to invoke slower relaxation if required.

In addition to the track swap out process, MediMusic provides evidence-based KPIs showing the service benefit and medication cost savings.

To find out more about MediMusic go to www.medimusic.co.

\section{Reference}

1. Guétin $S$, Giniès $P$, Siou D K A et al. The effects of music intervention in the management of chronic pain: a single-blind, randomized, controlled trial. Clin J Pain 2012; 28: 329-337.

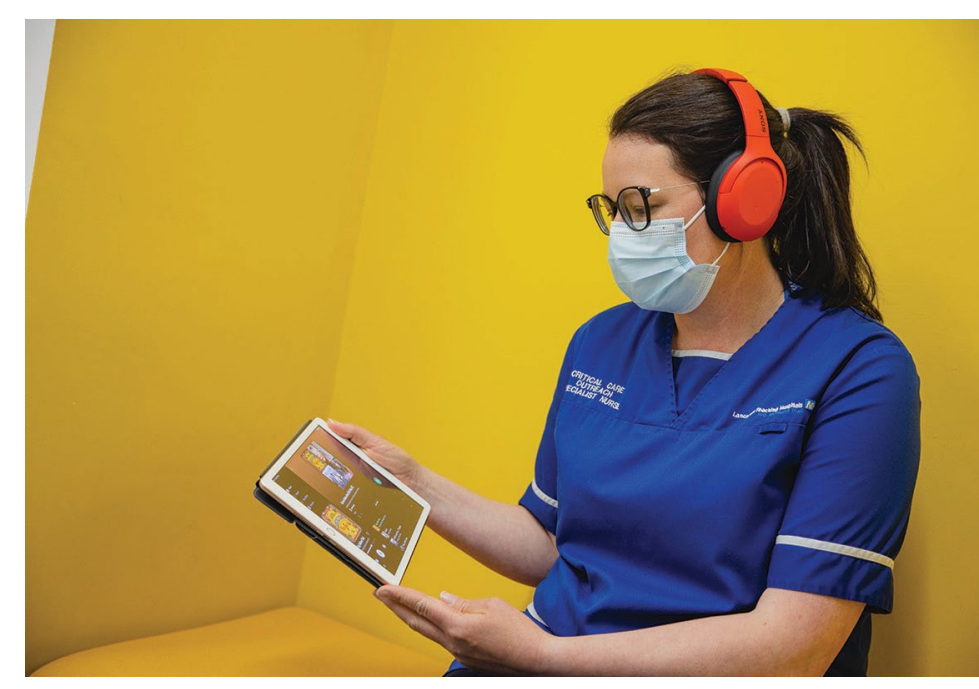

